\author{
Amalia Donia Sofio \\ Franco Mazzuca \\ Francesco S. Mennini
}

\section{General disease costing principles}

\author{
A.D. Sofio • F.S. Mennini ( $\square$ ) \\ CEIS-Health Economics Research \\ Department, \\ Faculty of Economics, \\ Tor Vergata University of Rome, \\ Via Columbia 2, I-00133 Rome, Italy \\ e-mail: f.mennini@uniroma2.it \\ Tel.: +39-06-72595642 \\ Fax: +39-06-233245536 \\ F. Mazzuca \\ Institute for Research on Population and \\ Social Policies, \\ National Research Council, Rome, Italy
}

\begin{abstract}
The term "disease costing" applies to those economic appraisal methodologies that ascertain the cost borne by a community because of a disease. This approach has been used to calculate the social and economic costs, in terms of loss of productivity, caused by a disease that only in the most serious cases results in the death of an individual. The production losses, also defined as indirect costs, are not the only social costs caused by diseases. In addition we point also to the negative variations that are reflected onto two fundamental quantities: consumption of resources and state of health. In addition to production losses and consumption of resources, the third group is unquestionably the most important in the healthcare sector, since it deals directly with variations in the state of
\end{abstract}

health. This group is denoted by the term "human costs". In the headache field, we are far behind other pathologies, where there is a greater knowledge of the economic aspects of both the pathology-related costs and the likely benefits resulting from different therapeutical approaches.

Notwithstanding the disease costing problems that are also reflected in the cost-benefit techniques, it is important for the economic analysis to gain ground since there is a growing need to keep account of the available resources and the results attainable in the healthcare policies, from the central to the peripheral levels, where the valuation tools prove even more expedient.

Key words Disease costing • Human capital $\cdot$ Health indicators
The term "disease costing" applies to those economic appraisal methodologies that ascertain the cost borne by a community because of a disease. The general principles that gave rise to disease costing originate from an attempt by William Petty to ascertain the social cost determined by the plague that had struck London in the second half of the seventeenth century. The method he used is commonly known as the human capital approach, and allows calculation of the economic contribution to production of an individual. In particular, the aim of this method of appraisal is to arrive at the explicit value to be attributed to human life and, therefore, the cost in terms of loss of productivity caused by death.
In a book dating back to 1699 [1], Petty attributed to human life a value that was equal to the capital amount that, invested at a given interest rate, would have yielded an income equal to the gross domestic product divided by the total number of inhabitants [2]. According to this British statesman, in 1676 nearly 200 thousand lives might have been saved - in the most optimistic hypothesis - thanks to the progress in medicine. Attributing a minimum value of 20 pounds to human life - this being the price of a slave on the London market - Petty estimated that the advantage which could have been gained in England by the developments of medicine amounted to 4 million pounds. 
More recently, this approach has been used to calculate the social and economic costs, in terms of loss of productivity, caused by a disease that only in the most serious cases results in the death of an individual. The distinction drawn by Mushkin [3] proved quite useful in this respect. In a theoretical study dealing with the human capital approach, the latter differentiated the production losses due to mortality from those resulting from morbidity, that is the non-fatal outcome of a disease. The production losses resulting from morbidity are reflected in work-time reductions that may be calculated through absence. Recent studies have demonstrated that, in EU countries, the working days lost as a result of migraine vary from 1.9 to 3.2 per patient per year. In all studies, regardless of nationality, women stay away from work more often than men $[4,5]$.

In practical terms, the market rate of wages is multiplied by the hours or days lost that, in case of death, turn into years of work lost. Such a method is also applied to activities for which there is no market, as is the case of housewives, for whom a "shadow" price is calculated having recourse to the wages of housemaids.

In addition to these two types of loss, there is also a third component that, although quite relevant, defies any easy estimation: the production capacity losses (debilities) witnessed when a sick individual goes to work. Reduced levels of effectiveness account for $70 \%$ of all the work lost. In fact, all studies agree that the majority of days lost are not due to absence but to a lower level of effectiveness while continuing to work during migraine attack. Although evaluation of loss of productivity linked to migraine is extremely subjective, the residual level of effectiveness calculated in the various studies is fairly similar, between $56 \%$ and $72 \%[4,6]$. Hence, the value that would be obtained from the calculation of just the first two types of production losses could lead to an underestimation of the health benefits resulting from a reduction of these losses.

This distinction proves quite useful in those pathologies - and this is indeed the case of headaches - that cause high economic costs that are not measurable merely in terms of mortality.

The production losses, also defined as indirect costs, are not the only social costs caused by diseases. Drummond, one of the best-known health economists, pointed also to the negative variations that are reflected onto two fundamental quantities: consumption of resources and state of health [7]. The former are also defined as direct costs. Patients in primary care with headache cost at least $87 \%$ more than their similar-age and same gender counterparts without headache [8]. Patients with migraine exact as much as 1.6-fold higher overall costs in comparison to patients without migraine [9]. Direct costs include all the healthcare costs caused by a disease, from its diagnosis to the patient's treatment and rehabilitation. Hence, this means the clinical and instrumental analyses called for by a diagnosis, the drugs, as well as any other therapeutical measure (e.g. surgical operation, radiotherapy) $[4,10]$. The resources include the services provided by the health personnel (physicians, nurses and other workers) and that part of overhead costs imputable to the disease. Hospitalisation costs universally represent only a very small portion of total migraine management costs: the rate of hospitalization is less than $10 \%$ and varies from $2 \%$ in Denmark to $7 \%$ in the USA.

These are the most easily identifiable costs. However it is still not possible to precisely quantify the direct costs of migraine because there is general agreement that a high percentage of migraneurs never consult a physician for their illness - between $19 \%$ and $44 \%$ in the various studies - and that only a small percentage - from $16 \%$ to $36 \%$ - regularly consults a physician [11]. They may be differentiated depending on who provides the resources: the healthcare service, regardless of the type of system existing in the various countries (national healthcare service, panel-based healthcare system, or health insurance), or else the patients and their relatives. Such a distinction proves useful as it permits to detect the differences existing between disease costing - this being an economic appraisal method - and the financial analyses that only consider those costs that affect the year's end figures of a business. Indeed, economic appraisal is a method for valuating social costs, regardless of whoever bears them.

The calculation of direct costs must also include a number of costs that are not related to healthcare, such as the time needed to get to the facilities providing healthcare services. They also include transportation costs, and waitingtime costs, provided that this time is not subtracted from the productive activity since, otherwise, this would lead to a duplication where it would be also calculated among production losses. In the case of a few pathologies, one must also include special diets, changes in one's home, in the case of a few home-delivered treatments, or in one's clothes, when a prosthesis or a different medical device is called for.

Unfortunately, the economic and financial approaches intermingle to such an extent that they are easily mixed up. For example, the fixed levy (ticket) that patients pay as a cost-sharing mechanism translates into income for the providers of the healthcare services. This important financial element is not relevant from an economic point of view, as one needs to calculate the value of the resources that are behind that service rather than to what extent the patient pays for that service. In fact, it is only a "transfer" of expenditure from the community to the individual or, in case of a reduction of the fixed levy, from the individual to the community. On the other hand, the activity rended by unpaid voluntary personnel is an important economic cost item that has no financial expression since it is not entered in the balance sheet. 
In addition to production losses and consumption of resources, the third group is unquestionably the most important in the healthcare sector, since it deals directly with variations in the state of health. This group is denoted by the term "human costs".

The fact that this group of variations represents a cost is a recent acquisition, when it became manifest that the disease or the death of an individual results in damage that may be expressed in economic terms, regardless of the lower production or the resources needed to treat a disease. In fact, the negative variations in the state of health are still defined by quite a few scholars as "intangible" costs, to point to the difficulty in calculating them, or even "incommensurable" costs, as if they were impossible to calculate.

If it is true that health is a "hardly" quantifiable element, as shown by the lack of a specific definition of health measurable in quantitative terms, it is nonetheless a fact that an effort has been made for a number of years to overcome this limit through suitable "health indicators". While the literature on this specific subject has become one of the richest ever, with the formulation of scales and indexes, these contributions seldom aim at providing a sound tool for economic analysis.

Indeed, the main object is to single out the manifold aspects that make up the definition of health provided by the World Health Organization, that is a state of absolute physical and psychological well-being, or else to measure the different levels of health, moving from the slighter to the most serious cases.

However, before spending a few words on these indicators, it would seem advisable to take a step backwards and look into history to single out in the death rates the first tool to express the variations in the state of health of a population, followed later on by the morbidity rates.

The first half of the twentieth century witnessed the development of a statistical tool that kept track not only of the event of death, but also of the age of death. This led to the definition of the "potential years of life lost" (PYLL) indicator, which took into account early death due to a few diseases with respect to the age of the statistical average life expectancy of a given population.

Further to the advances in medicine and the consequent shift from infectious to chronic-degenerative pathologies, people realized that this tool was not suitable for measuring the effects on health expressed not only in its quantitative contents but also in qualitative terms.

From this point of view, the indicator that aroused the greatest interest was developed in the 1970s with the name of quality adjusted life years (QALYs). Thanks to this indicator, the individual years of life were calculated adopting different "weighting" in relation to the quality of life. In fact, a year of life of a disabled person may not be valued in the same way as a year of life of a healthy person.
The limit to this tool, which also exists in the PYLL, is an ethical limit and stems from the difficulty in comparing costs resulting from pathologies that affect individuals of differing age. The human costs for diseases that affect senior citizens could prove lower than those affecting children, the individuals involved being equal.

In the face of this limit, however, there is an unquestionable advantage: the possibility of expressing with a single indicator both the mortality-related costs and the costs resulting from a permanent or temporary disease-related disability. This is feasible when a scale is developed starting from states of health that may be likened to death and going up, through intermediate states of health, to the state of "well-being". While refraining from going into this subject, it should only be noted that a few tools, like Rosser's scale, also contain situations where death would be preferable, but this debate would lead us beyond the bounds of this report.

Conceptually similar to the QALYs, the disability adjusted life years developed a few years later have met with the approval of both the World Bank and the World Health Organization. Apart from a few differences that are not going to be dealt with, this index offers more equitable measures in respect of different age classes.

In any event, the recourse to health indicators for the evaluation of the state of health and its variations is quite recent. In addition to the previously mentioned human capital approach, there is a different method of expressing valuations relative to the state of health, meaning the "willingness to pay".

This concept was introduced by Jules Dupuit halfway through the nineteenth century with respect to the underestimation of public works that resulted from a valuation based on the national revenue receipts. This is why he introduced the willingness to pay as a higher value, matched in the opposite direction by a "willingness to receive", meaning compensation in case of damages caused by a public work.

Applying this method to the case of health, it may be formulated as follows: what amount would you be willing to pay to cut down your death risk by a given percentage?

Just like the human capital approach, even this method leads to results expressed in terms of money, and this ended up giving rise to quite a number of objections. The latter are connected with the difficulty of attributing a value to pain, stress and other sufferings caused by diseases to individuals and their relatives and friends, in order to get to the value of human life itself.

As previously pointed out, while the disease costing approach gives rise to different valuation methods from the very quantification of the cost elements, it becomes even more complex during the monetary evaluation phase. This relates not only to the state of health but also to the other two types of cost. With reference to production losses, the problem is not merely the evaluation of the activities that have no 
market, or the productive capacity losses that result when a sick worker actually goes to work. The problem is also connected with the fact that absences do not always turn into production losses. In the more serious case of death, from a collective rather than an individual point of view, there is the possibility of replacing the worker with an unemployed person, but even in case of a temporary absence there is the possibility of the activity not being strictly required.

Besides, insofar as resources are concerned, we are faced with the problem of whether the market price is the best indicator of the "opportunity cost", i.e. the expression of the real value in a competitive market. The markets, however, and the healthcare market in particular, are affected by a series of distortions (suffice it to consider, for instance, the presence of a sole purchaser represented by the national healthcare service, defined as a case of monopsony), or else oligopolies in the supply of a few goods and services.

These aspects, dealt with at length by the economic literature, are compounded by the problem of a few heavily taxed goods, such as fuels. In case of treatments provided at the patient's home, or other measures that call for a considerable expenditure with respect to this type of product, from an economic point of view taxes and duties should be subtracted from the related costs. This, however, would result in a considerable difference in respect of the financial analysis, which offers the dimension of the actual possibility of developing a few healthcare programs.

It may be noted that there are still many unsolved problems in disease costing, to the point that it still appears as a set of methods that may lead to extremely different out- comes depending on the valuation approach being used. Notwithstanding the above, it still represents an important tool in the healthcare-planning phase, as it permits to get to an initial value that may be used first of all in the relative evaluation, i.e. when the costs of different diseases are compared. In any event, it should be noted that, in this context, one needs to take into account not only the social cost of a given disease but also the real possibilities of cutting down those costs thanks to the existing technologies, meaning the diagnostic, therapeutic and rehabilitative tools that are already available or being adopted.

Hence, disease costing may be a preliminary appraisal with respect to subsequent cost-benefit analyses meant in a broad sense, i.e. including the cost-benefit analysis (CBA) proper together with the cost-effectiveness analysis (CEA) and the cost-utility analysis (CUA), and mostly the latter, as they are methodologies extensively used in the healthcare field.

By way of conclusion, it should be recalled that in the headaches field we are far behind other pathologies, where there is a greater knowledge of the economic aspects of both the pathology-related costs and the likely benefits resulting from different therapeutical approaches.

Notwithstanding the disease costing problems, which are also reflected in the cost-benefit approaches, it is important for the economic analysis to gain ground since there is a growing need to keep account of the available resources and the results attainable in the healthcare policies, from the central to the peripheral levels, where the valuation tools referred to above prove even more expedient.

\section{References}

1. Mooney GH (1977) The valuation of human life. MacMillan, London, p 54

2. Petty W (1699) Political arithmetic, or discourse concerning the extent and value of lands, people, buildings, etc. Clavel, London

3. Mushkin S (1973) Health as an investment. In: Cooper MH, Culyer EJ (eds) ...

4. Cerbo R, Pesare M, Aurilla C, Rondelli V, Barbanti P (2001) Socio-economic costs of migraine. J Headache Pain 2:S15-S19
5. Stewart WF, Celentano DD, Linet MS (1989) Disability, physician consultation, and use of prescription medication in a population-based study of headache. Biomed Pharmacother 43:711-718

6. Clarke CE, MacMillan L, Sondhi S, Wells NE (1996) Economic and social impact of migraine. Q J Med 89:77-84

7. Drummond MF (1980) Principles of economic appraisal in health care. Oxford University, Oxford

8. Fishman P, Von Korff M, Lozano P et al (1997) Chronic care costs in managed care. Health Affairs 16(3):239-247
9. Clouse JC, Osterhaus JT (1994) Healthcare resource use and costs associated with migraine in a managed healthcare setting. Ann Pharmacother 28(5):659-664

10. Hu XH, Markson LE, Lipton RB, Stewart WE, Berger ML (1999) Burden of migraine in the United States. Disability and economic costs. Arch Intern Med 159:813-818

11. Edmeads J, Findlay H, Tugwell P, Pryse-Phillips W, Nelason RF, Murray TJ (1993) Impact of migraine and tension-type headhache on life-style, consulting behaviour, and medication use: a Canadian population survey. Can J Neurol Sci 20:131-137 